\title{
Intervenções precoces na infância: observando a relação mãe-bebê em um serviço de saúde
}

\author{
Early intervention in childhood: watching the mother-baby relationship in a health service \\ Intervenciones precoces en la infancia: observando la relación madre-bebé en un servicio \\ de salud
}

\author{
Dorian Mônica Arpini* \\ Edinara Zanatta ${ }^{* *}$ \\ Rafaela Quintana Marchesan ${ }^{* * *}$ \\ Sabrina Dal Ongaro Savegnago ${ }^{* * * *}$ \\ Pedro Henrique Bernardi****
}

\begin{abstract}
Resumo
Este artigo tem como objetivo apresentar as atividades desenvolvidas em um projeto de extensão. Tal projeto visa a observar a relação entre crianças de 0 a 2 anos e suas mães nos atendimentos realizados pelo Programa da Criança em uma Unidade Básica de Saúde, com o intuito de detectar possíveis riscos ao desenvolvimento infantil de modo precoce. Utilizase da técnica de observação, tendo por base os Indicadores Clínicos de Risco ao Desenvolvimento Infantil (IRDI). Caso se identifiquem riscos ao desenvolvimento, são utilizados outros recursos técnicos que podem incluir entrevistas de orientações aos familiares e encaminhamentos à rede de atendimentos especializados. Os resultados alcançados indicam a importância do vínculo entre a equipe e os usuários, a maior atenção às díades e suas relaçóes afetivas e a possibilidade de detecção precoce das problemáticas envolvendo os aspectos emocionais. Por fim, destaca-se a ação interdisciplinar e a atuação da Psicologia na promoção de saúde.
\end{abstract}

Palavras-chave: Relações familiares. Desenvolvimento infantil. Saúde pública. Promoção da saúde. Relação mãe-filho.

\footnotetext{
Texto recebido em fevereiro 2013 e aprovado para publicação em maio de 2014.

*Doutora em Psicologia Social pela Pontifícia Universidade Católica de São Paulo, Professora Associada do Programa de PósGraduação em Psicologia da Universidade Federal de Santa Maria. Endereço: Rua Tiradentes, no 23, ap. 701 - Centro, Santa Maria-RS. CEP: 97050-730. E-mail: monica.arpini@gmail.com.

"Mestranda do Programa de Pós-Graduação em Psicologia da Universidade Federal de Santa Maria. Endereço: Rua Serafim Valandro, no 464, ap. 203/A - Centro, Santa Maria-RS. CEP: 97015-631.E-mail: edi.zanatta@hotmail.com.

*** Pós-graduanda do Programa de Residência Multiprofissional Integrada em Saúde Mental no Sistema Público de Saúde da Universidade Federal de Santa Maria. Endereço: Rua Alberto Pasqualini, no 287 - Centro, Restinga Seca-RS. CEP: 97200-000. E-mail: rafaelamarchesan@hotmail.com.

${ }^{* * *+}$ Mestra em Psicologia pela Universidade Federal de Santa Maria, doutoranda do Programa de Pós-Graduação em Psicologia da Universidade Federal do Rio de Janeiro, bolsista CNPq. Endereço: Praia de Botafogo, no 356, ap. 329 - Botafogo, Rio de Janeiro-RJ. CEP: 22250-040. E-mail: binasavegnago@yahoo.com.br.

${ }^{* * *+*}$ Acadêmico do Curso de Psicologia da Universidade Federal de Santa Maria, bolsista FIEX UFSM 2012. Endereço: Rua Coronel Niederauer, no 1440, ap. 6 - Centro, Santa Maria-RS. CEP: 97015-122. E-mail: phob13@hotmail.com.
} 


\begin{abstract}
This article aims to present the activities developed in an extension Project. Such a project aims to observe the relationship between children, from zero to two years old, and their mothers, in the assistance carried out in the Child Program in a Basic Health Unit, targeting to detect possible early risks to child development. Observation techniques are used, based on the Clinical Risk Indicators in Child Development (CRICD). If risks to development are identified, other technical resources are used, that may include orientation interviews of family members and redirections to specialized services network. Results indicate the importance of the bond between the health care team and the users, the need of greater care regarding dyads and their relationships and the possibility of early detection of problems involving emotional aspects. Finally, the interdisciplinary actions and the role of Psychology concerning health promotion are highlighted.
\end{abstract}

Keywords: Family relations. Child development. Public health. Health promotion. Mother-child relations.

\title{
Resumen
}

Este artículo tiene como objetivo presentar las actividades desarrolladas en un proyecto de extensión. Este proyecto pretende observar la relación entre niños de cero a dos años y sus madres en la atención realizada por el Programa Infantil de una Unidad Básica de Salud, con el fin de detectar precozmente los posibles riesgos para el desarrollo del niño. Se utiliza la técnica de observación basada en los Indicadores Clínicos de Riesgo al Desarrollo Infantil (IRDI). Cuando se identifican riesgos para el desarrollo, otros recursos técnicos son utilizados, que pueden incluir entrevistas de orientación a los familiares y derivaciones a la red de atención especializada. Los resultados obtenidos indican la importancia del vínculo entre el equipo y los usuarios, una mayor atención a las duplas y sus relaciones afectivas y la posibilidad de detección precoz de los problemas que afectan a los aspectos emocionales. Por último, se destaca la acción interdisciplinaria y la actuación de la Psicología en la promoción de la salud.

Palabras clave: Relaciones familiares. Desarrollo infantil. Salud pública. Promoción de la salud. Relaciones madre-hijo. 


\section{Introdução}

B aseados no relatório da Organização Mundial de Saúde (Organização Mundial da Saúde [OMS], 2001) e em outros estudos, Lerner e Kupfer (2008) e Kupfer et al. (2009) expõem que os transtornos mentais são comuns na infância e adolescência, porém o diagnóstico e o tratamento são insuficientes. Mesmo sendo problemas relevantes para o desenvolvimento das crianças, não têm recebido atenção adequada e suficiente das políticas públicas, o que acaba deixando um grande número de crianças sem assistência adequada. Nesse sentido, o projeto de extensão "Intervençôes precoces na infância: um projeto junto ao Programa da Criança de uma Unidade Básica de Saúde" foca na prevenção primária, a qual compreende a promoção de saúde (Arpini \& Santos, 2007; Westphal, 2006) e a prevenção de doenças, visto que há a possibilidade de haver a detecção precoce de possíveis riscos ao desenvolvimento, além do olhar sobre o bem-estar das crianças.

Desse modo, desenvolvem-se ações com foco nas relações familiares e se observa se, na relação entre mãe e filho, estão presentes aspectos que indiquem riscos ao desenvolvimento infantil, valendo-se para tanto dos Indicadores Clínicos de Risco ao Desenvolvimento Infantil (IRDI) para as crianças de 0 a 18 meses. Tal instrumento foi elaborado com base na teoria psicanalítica, por meio de uma pesquisa multicêntrica desenvolvida pelo GNP (Grupo Nacional de Pesquisa), um grupo de psicanalistas reunidos, em 1999, pelo Ministério da Saúde, no período entre 2000 e 2008, e que resultou no instrumento composto por 31 indicadores clínicos de risco psíquico ou de problemas de desenvolvimento infantil observáveis nos primeiros 18 meses de vida de uma criança (Kupfer et al., 2008; Jerusalinsky et al., 2009).

Na referida pesquisa, desenvolvida pelo GNP, privilegiou-se a articulação entre desenvolvimento e sujeito psíquico, sem desconsiderar os processos maturativos de ordem neurológica e genética; estes geralmente alvos das pesquisas sobre desenvolvimento. O sujeito psíquico é concebido por Jerusalinsky et al. (2009) como um elemento organizador do desenvolvimento, tanto na esfera física, psicomotora, cognitiva e psíquica, o que faz com que os responsáveis por seus cuidados e por sua evolução tenham um papel primordial, já que o lugar do sujeito dependerá das ações gerais que o cuidador realizará na primeira infância. Assim, se os processos de formação não forem governados pelos outros que rodeiam a criança, o lugar de sujeito corre o risco de não se constituir. 
Como exposto acima, na construção dos indicadores da pesquisa IRDI, os aspectos ligados ao sujeito psíquico foram privilegiados, de modo que a presença dos indicadores na criança aponta que ela está em desenvolvimento saudável, e a sua ausência é que indica riscos ao desenvolvimento. Dessa forma, os indicadores foram concebidos de maneira positiva, de modo que, quando incluídos em um protocolo de consultas regulares de pediatras, estes verão a saúde da criança, desfocando o olhar sobre a doença (Kupfer et al., 2008; Jerusalinsky et al., 2009). Assim, caso haja a ausência de algum IRDI, pode-se suspeitar de que algo não está bem, buscando-se intervir precocemente sobre esse aspecto, a fim de orientar a mãe sobre sua maternagem.

No entanto, em um segundo momento, esse instrumento passou por uma revisão que levou à redução do número de indicadores, passando para 18. A redução se deu a partir da observação de que alguns indicadores se mostraram mais sensíveis, apresentando maior poder preditivo para risco psíquico. Dessa forma, o instrumento validado com 31 indicadores apresenta capacidade preditiva para problemas de desenvolvimento, mas não tão específicas para risco psíquico (Kupfer et al., 2008). ${ }^{1}$

Quanto aos aspectos físicos do desenvolvimento, Winnicott(1999) coloca que não podemos ver um bebê somente por esse ângulo, já que, segundo o autor, o bebê "é mais que sangue e ossos" (p. 20). Para o estudioso, o desenvolvimento da saúde mental do indivíduo está sendo construído pela mãe, a qual proporciona um ambiente facilitador para o bebê, assentando as bases da saúde mental do filho.

De acordo com Winnicott (1983), o bebê só se torna um sujeito se estiver envolvido ao cuidado materno, visto que é totalmente dependente, e sem essa condição não pode começar a vir a ser. A criança começa a ser a partir de um cuidado satisfatório, proporcionado por uma mãe que provê um ambiente suficientemente bom, capaz de auxiliar o bebê a alcançar as satisfações, ansiedades e conflitos inatos a cada etapa (Flores, Ramos, Moraes \& Beltrami, 2013).

As mães psiquiatricamente saudáveis se preparam, nos últimos meses de gravidez, para se colocar no estado de realizar a tarefa especializada que terão de cumprir, voltando ao estado normal nas semanas e meses após o nascimento. É o estado de identificação com o bebê que as tornam capazes de se colocarem no lugar dos filhos, indo ao encontro das necessidades básicas destes, de uma forma que não pode ser imitada e nem ensinada (Winnicott, 1999).

1 Para maior detalhamento em relação ao IRDI, consultar ; Jerusalinsky et al. (2009) e Lerner \& Kupfer (2008). 
Segundo esse mesmo autor, esse estado de sensibilidade exacerbada é quase uma doença, porém a mãe se recupera dele, sendo esse o estado muito especial que ele chama de Preocupação Materna Primária. Nesse período, a mãe preocupase com o seu bebê e em atender e adaptar-se às necessidades dele, excluindo de forma temporária e normal qualquer outro interesse que poderia vir a ter. Porém não são todas as mães que conseguem se identificar com o bebê nessa fase inicial do desenvolvimento, não contraindo essa "doença normal" (Winnicott, 2000; Beltrami, Moraes \& Ramos, 2013).

Entretanto, quando a mãe é suficientemente boa, a linha da vida de uma criança é muito pouco perturbada por reaçôes a intrusōes, pois ela consegue uma adaptação sensível às necessidades do bebê, colocando-se no lugar deste, e assim assenta as bases para um desenvolvimento saudável. Dessa forma, "capacita o bebê a começar a existir, a ter experiências, a construir um ego pessoal, a dominar os instintos e a defrontar-se com todas as dificuldades inerentes à vida" (Winnicott, 2000, p. 404).

Nesse sentido, Klein (1991) coloca que, a partir do vínculo que o bebê estabelece com a mãe, centrado na relação com o seio bom, as ansiedades suscitadas pelo processo de nascimento e pela necessidade de adaptação às condições novas são aliviadas. Assim, ao sentir o amparo, o conforto e, principalmente, a gratificação em receber o alimento e sugar o seio, o bebê inicia-se na relação com a mãe "boa" (p. 121).

Segundo Bowlby (2002), desde os primeiros dias de vida, os bebês desfrutam da companhia humana, ficando calmos quando em interação social e até parecendo sentir prazer em observar as pessoas à sua volta. Os bebês sentem amor por suas figuras de apego, ficando alegres com a chegada destas, e sentem-se seguros com a presença ou proximidade da figura principal de apego. Contudo uma ameaça de perda gera grande ansiedade, e a perda real suscita uma tristeza profunda.

Entende-se, assim, que há um vetor indo da mãe para o filho e um vetor indo do filho para a mãe, havendo uma troca afetiva recíproca. A presença da mãe, inclusive sua própria existência, suscita reações no bebê, e, igualmente, a presença e existência do bebê evocam reaçóes na mãe. Dessa forma, ações realizadas pelo filho que a mãe sente como agradáveis são encorajadas, e serão facilitadas por ela, que terá uma influência direta no desenvolvimento do bebê através das suas preferências, de modo que "seus afetos, seu prazer, suas próprias ações, conscientes ou inconscientes, facilitam inúmeras e várias ações do filho" (Spitz, 1998, p. 125; Beltrami et al., 2013). 
Nesse sentido, este artigo tem como objetivo divulgar as atividades de um projeto de extensão realizado junto ao Programa da Criança de uma unidade básica de saúde, que atenta e intervém sobre a relação mãe-filho (ou quem cumpra a função materna), e que tem como intuito qualificar essa relação e detectar possíveis riscos ao desenvolvimento infantil de modo precoce. Pretende-se que esta experiência seja compartilhada por outros profissionais da Psicologia e da área da saúde, estimulando práticas de cuidado focadas na prevenção primária.

\section{Caracterização}

O Programa de Assistência Integral à Saúde da Criança (PAISC) foi uma das estratégias nacionais adotadas, visando a incrementar a atenção dos serviços de saúde, identificando e priorizando ações básicas que fossem eficazes no controle dos problemas de saúde e envolvessem baixa complexidade tecnológica (Brasil, 1986). No caso da assistência infantil, tais ações foram denominadas "Ações Básicas na Assistência Integral à Saúde da Criança”, devendo constituir o núcleo da assistência prestada na rede básica de serviços de saúde (Arpini \& Santos, 2007).

O Ministério da Saúde contou com o apoio do UNICEF e da Organização Pan-Americana da Saúde, e previu cinco ações básicas: acompanhamento do crescimento e desenvolvimento; aleitamento materno e orientações para o desmame; controle de doenças diarreicas; controle de infecções respiratórias agudas e controle de doenças preveníveis por imunização. Para tanto, foram distribuídos pela rede de atenção à saúde manuais que procuravam definir e justificar o conteúdo das referidas ações, normatizando seus procedimentos e padronizando as condutas que delas decorrem (Brasil, 1987).

O período do nascimento aos 5 anos de idade é muito importante para o crescimento e desenvolvimento, devido à alta velocidade, ritmo, intensidade e vulnerabilidade do processo. Vários países vêm acompanhando o crescimento e o desenvolvimento de suas crianças, e percebem diversas vantagens: é uma metodologia universal, culturalmente aceitável, simples, econômica e não traumática, o que permite identificação de crianças em situação de risco, promovendo, além de uma intervenção precoce, a garantia de recursos para grupos prioritários (Brasil, 1986).

O acompanhamento do crescimento e desenvolvimento é realizado com base em ações de saúde, realizadas numa frequência mínima de observações e avaliações, para melhor assistir o processo de cada criança. Entre as ações, realizase: 

a) obtenção de dados do processo;
b) registro e interpretação das informações de acordo com padrão preestabelecido;
c) busca de explicações para as eventuais anormalidades ou deficiências detectadas; $\mathrm{e}$
d) assistência do processo de crescimento, mantendo as condições favoráveis ou possibilitando a correção de fatores que venham a comprometer o desenvolvimento saudável.

Recentemente, foi lançado pelo Ministério da Saúde o Programa Rede Cegonha, o qual propõe melhorias no atendimento às mulheres durante a gravidez, parto, pós-parto e ao recém-nascido, incluindo também ações para as crianças até os 2 anos de idade, visando ao crescimento e ao desenvolvimento saudável destas (Portaria no 1.459, de 24 de junho de 2011). Também nessa direção, pode-se fazer referência ao Programa Primeira Infância Melhor (PIM), proposto pelo Estado do Rio Grande do Sul com o objetivo de orientar as famílias a promoverem o desenvolvimento integral das crianças até os 6 anos de idade (Lei Estadual no 12.544, de 3 de julho de 2006).

\section{Métodos da ação}

A ação é realizada junto a uma unidade de atenção básica de uma cidade de porte médio do interior do Rio Grande do Sul. O projeto de extensão, registrado na instituição de ensino sob o número 029747, desenvolve suas atividades junto ao Programa da Criança, sendo a ação extensionista realizada por alunos de graduação e pós-graduação em Psicologia da Universidade Federal de Santa Maria (UFSM). Cabe ressaltar que, antes de iniciarem as práticas extensionistas propriamente ditas, esses alunos reuniram-se em um grupo de estudos, juntamente com o professor orientador, a fim de se capacitarem para a prática, a partir da leitura e discussão de textos e da apropriação do instrumento IRDI. Destaca-se, ainda, que no Programa atendem profissionais e alunos do curso de Enfermagem da UFSM, de modo que as ações são realizadas por uma equipe interdisciplinar.

No que concerne ao funcionamento do Programa da Criança, este presta atendimento durante três turnos da semana, de modo que os alunos de graduação e pós-graduação em Psicologia que desenvolvem a ação extensionista distribuamse entre esses turnos, a fim de possibilitar a maior cobertura possível ao Programa. O projeto de extensão junto ao referido Programa está em funcionamento desde maio de 2011, e os dados aqui apresentados compreenderão os atendimentos prestados no período de 2011 e 2012. 
Quanto às ações desenvolvidas pelo Programa, estas incluem o agendamento prévio das crianças, de modo que a agenda prevê seis atendimentos por turno, com duração de cerca de 30 minutos por consulta. Nessas consultas, estão incluídas as ações de Enfermagem, a saber: peso, medidas, controle da caderneta de vacinas e orientações quanto à alimentação, higiene e cuidados com o bebê. À Psicologia, por sua vez, cabe o cuidado aos aspectos relacionais envolvendo as trocas entre o bebê e seus cuidadores.

No que se refere ao método, o recurso técnico utilizado pela equipe de Psicologia é prioritariamente a observação, a qual se mostra importante quando se busca conhecer e compreender o desenvolvimento inicial, sobretudo os aspectos que envolvem a dinâmica relacional. Essa observação é realizada na sala de atendimento do Programa da Criança, sendo norteada pelo instrumento elaborado por Jerusalinsky et al. (2009), denominado "Indicadores Clínicos de Risco ao Desenvolvimento Infantil" (IRDI), o qual compreende a faixa etária dos 0 aos 18 meses. Cabe destacar, ainda, que o uso do protocolo fica aos cuidados da equipe de Psicologia. Desse modo, estando um ou mais indicadores ausentes, é possível que se pensem formas de intervenção precoces, na tentativa de se evitar que os riscos se tornem problemas mais sérios e que comprometam o crescimento saudável dos bebês. Assim, se a partir das observações, algum risco para o desenvolvimento infantil é identificado, os casos são levados para as supervisões acadêmicas, a fim de que se promovam a discussão e a definição de possíveis intervenções ou encaminhamentos, buscando assim desenvolver o projeto da forma mais efetiva e coerente com a realidade local (Oliveira, Gabarra, Marcon, Silva \& Macchiaverni, 2009).

Podem ser utilizados, além disso, outros recursos técnicos:

a) entrevistas com familiares, com o intuito de se conhecer mais sobre a dinâmica e a realidade familiar, assim como as dificuldades e facilidades encontradas por esse grupo para desempenhar as funçôes de maternagem e paternagem;

b) orientações aos familiares da criança, com o objetivo de promover relações cuidador-criança, comportamentos e hábitos mais saudáveis para o desenvolvimento infantil; e

c) encaminhamentos para outros profissionais e serviços de saúde, caso seja detectada a necessidade da criança ou familiar realizar alguma avaliação ou tratamento, sendo realizados, dessa forma, encaminhamentos para pediatra, neurologista, CAPS Infantil, Ambulatório de Saúde Mental, entre outros. 
Além disso, quando necessário, os casos podem ser levados para discussão em um grupo interdisciplinar denominado "Detecção e Estimulação Precoces: uma Perspectiva Interdisciplinar", formado por acadêmicos e profissionais das áreas de Psicologia, Fonoaudiologia, Fisioterapia e Terapia Ocupacional, para que se efetue um planejamento terapêutico singular. $\mathrm{O}$ referido grupo interdisciplinar trabalha, assim, no sentido de detectar e tratar problemas no desenvolvimento infantil, seja nos aspectos psicomotores, cognitivos, psíquicos ou linguísticos.

\section{Resultados}

No que se refere à situação do atendimento, as crianças geralmente comparecem acompanhadas de suas mães, sendo pouco frequente a presença dos pais ou outro familiar no Programa. Além das ações desenvolvidas pela equipe interdisciplinar já citadas anteriormente, a equipe presta todo o amparo em relação às angústias, medos e dúvidas que surgem do papel de ser mãe. Destaca-se como importante o dizer de Arpini e Santos (2007), ao referirem que, na maioria das vezes, as perguntas são dirigidas à equipe de Enfermagem e referem-se a aspectos do desenvolvimento físico. Acrescentam, porém, que foi "por meio deles que se construiu o caminho para a discussão e o fortalecimento do aspecto relacional, das trocas que ocorrem entre a mãe/familiar e a criança e do lugar que esse novo ser ocupa no âmbito familiar" (p. 159).

Nesse sentido, procura-se observar como está o vínculo da díade mãe-filho (ou cuidador) e como a mãe está desempenhando o seu papel nessa relação, a fim de se entender o ambiente e a dinâmica familiar onde essa criança está inserida, com o intuito de se realizarem orientaçôes mais adequadas à realidade familiar. Em concordância com essa proposta, Serralha (2013) aponta para a importância da ampliação dos cuidados que têm sido oferecidos nos serviços públicos, os quais têm se caracterizado pela articulação e integração dos cuidados oferecidos, abarcando a mãe e a relação mãe-bebê. Nas palavras de Arpini e Santos (2007), a questão socioeconômica deve ser considerada, de modo que é muito importante que os profissionais do serviço conheçam, primeiramente, a realidade em que vive a família, a fim de que não se forneçam orientações difíceis de serem seguidas ou que possam constranger o cuidador.

Quanto à frequência dos atendimentos, estes são geralmente agendados mensalmente, exceto quando se verifica a necessidade de alguma criança retornar antecipadamente. Cabe ressaltar, ainda, que houve o não comparecimento de alguns usuários na data marcada, notadamente daquelas crianças que estariam participando do Programa pela primeira vez. Desse modo, evidenciou-se que, a partir do momento em que o vínculo entre a equipe e a díade mãe- 
criança é estabelecido, tornam-se mais escassas as faltas ao horário agendado, o que pode ser entendido positivamente como a satisfação das mães quanto ao acompanhamento que têm recebido.

No que diz respeito ao método utilizado, especificamente ao protocolo IRDI, têm-se identificado algumas dificuldades na observação de aspectos que constam no instrumento. Isso se deve ao fato de que, por vezes, os indicadores que compõem o instrumento não são possíveis de serem detectados na situação do atendimento, por exemplo:

a) a criança aceita alimentação semissólida, sólida e variada;

b) a mãe propõe algo à criança e aguarda sua reação;

c) a criança busca ativamente jogos e brincadeiras amorosas com a mãe; e

d) a mãe alterna momentos de dedicação à criança com outros interesses. Com relação a essa dificuldade, a equipe busca sanar essa lacuna por meio do diálogo com as mães, indagando a respeito dos aspectos que não estão sendo observados e que são importantes de serem avaliados.

Além disso, os resultados destacam o saber que a mãe porta em relação ao seu bebê, conforme atribui e reconhece, no filho, necessidades, preferências, características, modos de agir e de se comunicar. Além disso, pôde-se perceber a existência de uma comunicação entre a díade, vivenciada de modo afetivo entre ambos. Pôde-se acompanhar que as vocalizaçóes, os gestos e as atitudes dos bebês eram respondidos pelas mães, na maioria das vezes, atribuindo-lhes um sentido. Os bebês, por sua vez, ao ouvirem a voz da mãe, sentirem o seu toque ou observarem a proximidade dela, acalmavam-se. Com isso, pôde-se perceber, de modo satisfatório, o desenvolvimento da criança e a relação mãe-bebê.

Ainda nos parece importante frisar que tais indicadores no âmbito da saúde pública podem, muitas vezes, passar despercebidos pelos profissionais de saúde que, de maneira geral, podem estar mais sensíveis aos aspectos físicos do desenvolvimento e crescimento, os quais historicamente constituem o foco dos serviços na área materno-infantil. Desse modo, os resultados sinalizam, para além da importância desse momento na constituição psíquica da criança, o lugar e a atenção atribuída às mães nesse momento, superando uma perspectiva de naturalização da maternidade, com vistas a construir uma prática que busca, no investimento e nos cuidados maternos, a matriz para a constituição psíquica, valorizando a sua presença e seu fazer nesse momento da vida do filho (GomesKelly, 2011; Serralha, 2013). 
Por meio da ação interdisciplinar que integra Enfermagem e Psicologia, temse inovado e ampliado as ações da Psicologia, sobretudo na promoção de saúde, aspecto certamente muito importante para a formação de novos profissionais, uma vez que estamos tratando de uma ação extensionista. Outro aspecto destacado por Blank (2003), citado por Santos, Resegue e Puccini (2012), é a compreensão de que a inserção na puericultura desse cuidado para as relaçóes familiares tem demonstrado "que a puericultura deixou de ser uma especialidade médica e passa a ser desenvolvida por uma equipe multiprofissional em parcerias, incluindo as famílias e as comunidades" (p. 163). Destaca-se, assim, a relevância das primeiras relações entre a criança e a mãe, e a importância de um serviço onde possam ser estimuladas e acompanhadas. Nesse sentido, o Programa da Criança, mediante a ação interdisciplinar, tem se constituído num espaço para o acompanhamento e fortalecimento de vínculos.

Destaca-se que tal intervenção está atingindo seus objetivos quanto à observação e intervenção junto à relação mãe-filho no período inicial do desenvolvimento, constituindo-se, portanto, como uma estratégia de detecção precoce de riscos ao desenvolvimento infantil, prevenção de doenças e promoção de saúde. Assim, como fim maior, tem-se obtido a melhoria da qualidade de vida e do bem-estar da população-alvo do projeto de extensão, a saber, a mãe (aqui entendida como a pessoa que exerce a função materna) e sua criança.

Por seus resultados positivos, o projeto de extensão está tendo continuidade junto ao Programa da Criança e recentemente ampliou suas atividades para o Programa dos Agentes Comunitários de Saúde vinculados à unidade na qual o projeto é desenvolvido. Tal ampliação tem-se realizado por meio de encontros mensais nos quais se discutem aspectos relacionados ao desenvolvimento infantil, ao instrumento IRDI e às relações familiares, além de outros temas de interesse e demanda local.

\section{Considerações finais}

Ressalta-se, por fim, a importância de se realizarem projetos que intervenham junto à infância, com o intuito de prestar a atenção adequada às questóes relacionadas ao desenvolvimento físico e emocional de crianças. Evidencia-se como fundamental ainda o trabalho interdisciplinar, posto que a saúde-doença é um fenômeno multideterminado, requisitando para tanto de um olhar que envolva vários saberes que, inter-relacionados, deem conta da complexidade de elementos e fatores que influenciam no desenvolvimento infantil. Tal ação destaca, ainda, a importância das relações estabelecidas entre a equipe e os usuários do serviço, a qual tem se pautado pelo acolhimento, interesse e sensibilidade com relação às demandas apresentadas. 
Não se pode deixar de assinalar a relevância da prática psicológica inserida nesse contexto, uma vez que a Saúde Pública ainda se constitui num campo de atuação novo para profissionais da Psicologia, exigindo a necessidade de qualificação dos profissionais para atender aos pressupostos do Sistema Único de Saúde.

\section{Referências}

Arpini, D. M. \& Santos, B. R. (2007). Programa da Criança: espaço de promoção de saúde e fortalecimento dos vínculos. Revista Psicologia Argumento, 25(49), 155-59.

Beltrami, L. Moraes, A. B. \& Ramos, A. P. (2013). Ansiedade materna puerperal e risco psíquico para o desenvolvimento infantil. Revista Distúrbios da comunicação, 25(2), 229-339.

Bowlby, J. (2002). Apego e perda: apego. São Paulo: Martins Fontes.

Brasil. Ministério da Saúde. INAN, Instituto Nacional de Alimentação e Nutrição. (1987). Programa Nacional de Aleitamento Materno. Brasília: Ministério da Saúde.

Brasil. Ministério da Saúde. SNPES/DINSAMI, Instituto Nacional de Assistência Médica da Previdência Social - INAMPS. (1986). Programa de Atenção Integral à Saúde da Criança, Acompanhamento e Desenvolvimento. Brasília: Ministério da Saúde.

Flores, M. R., Ramos, A. P., Moraes, A. B. \& Beltrami, L. (2013). Associação entre índices de risco ao desenvolvimento infantil e estado emocional materno. Revista CEFAC, 15(2), 348-360.

Gomes-Kelly, R. E. O. (2011). Intervenção precoce: prevenção, tratamento, profilaxia? Reflexões sobre o atendimento às crianças pequenas e a saúde mental na infância. In M. C. Laznik \& D. Cohen (Eds.), O bebê e seus intérpretes: clínica e pesquisa (pp. 171-178). São Paulo: Instituto Langage.

Klein, M. (1991). Inveja e gratidão: e outros trabalhos. Rio de Janeiro: Imago.

Kupfer, M. C. M., Bernardino, L. M. F., Jerusalinsky, A. N., Rocha, P. S., Lerner, R. \& Pesaro, M.E. (2008). A pesquisa IRDI: resultados finais. In R. Lerner \& M. C. M. Kupfer (Orgs.), Psicanálise com crianças: clinica e pesquisa (pp. 221230). São Paulo: FAPESP, Escuta. 
Jerusalinsky, A., Kupfer, M.C., Bernardino, L. F., Wanderley, D., Rocha, P., Molina, S., ... Lerner, R. (2009). Valor preditivo de indicadores clínicos de risco para o desenvolvimento infantil: um estudo a partir da teoria psicanalítica. Revista Latino-americana de Psicopatologia Fundamental, 6(1), 48-68.

Lei Estadual no 12.544, de 3 de julho de 2006. (2006, 3 de julho). Institui o Programa Primeira Infância Melhor e dá outras Providências. Porto Alegre: Governo do Estado do Rio Grande do Sul. Recuperado a partir de http://www. pim.saude.rs.gov.br/a_PIM/legislacao/LeiDoPIM-12544Pagina1.pdf e http:// www.pim.saude.rs.gov.br/a_PIM/legislacao/LeiDoPIM-12544Pagina2.pdf

Lerner, R. \& Kupfer, M. C. M. (2008). Psicanálise com crianças: clinica e pesquisa. São Paulo: Fapesp, Escuta.

Oliveira, L. D. B., Gabarra, L. M., Marcon, C., Silva, J. L. C. \& Macchiaverni, J. (2009). A brinquedoteca hospitalar como fator de promoção no desenvolvimento infantil: relato de experiência. Revista Brasileira de Crescimento e Desenvolvimento Humano, 19(2), 306-312.

Organização Mundial da Saúde (2001). Relatório sobre a saúde no mundo: saúde mental: nova concepção, nova esperança. Recuperado a partir de http://www. abebe.org.br/wp-content/uploads/oms2001.pdf

Portaria $n^{\circ}$ 1.459, de 24 de junho de 2011. (2011, 24 de junho). Institui, no âmbito do Sistema Único de Saúde - SUS - a Rede Cegonha. Diário Oficial da União, Brasília. Recuperado a partir de http://bvsms.saude.gov.br/bvs/ saudelegis/gm/2011/prt1459_24_06_2011.html

Santos, R. C. K., Resegue, R. \& Puccini, R. F. (2012). Puericultura e a atenção à saúde da criança: aspectos históricos e desafios. Revista Brasileira de Crescimento e Desenvolvimento Humano, 22(2), 160-165.

Serralha, C. A. (2013). A ética do cuidado e as ações em saúde e educação. In Z. Loparic (Ed.), Winnicott e a ética do cuidado (pp. 319-338). São Paulo: DWW.

Spitz, R. A. (1998). O primeiro ano de vida. São Paulo: Martins Fontes.

Westphal, M. F. (2006). Promoção da saúde e prevenção de doenças. In G. W. S. Campos, M. C. S. Minayo, M. Akerman, M. Drumond Júnior \& Y. M. Carvalho (Eds.), Tratado de Saúde Coletiva (pp. 635-67). São Paulo: Hucitec; Rio de Janeiro: Fiocruz. 
Winnicott, D. W. (1983). O ambiente e os processos de maturação: estudos sobre a teoria do desenvolvimento emocional. Porto Alegre: Artes Médicas.

Winnicott, D. W. (1999). Os bebês e suas mães. São Paulo: Martins Fontes.

Winnicott, D. W. (2000). Da pediatria à psicanálise: obras escolhidas. Rio de Janeiro: Imago. 